IFUP-TH 54/92

hep-lat/9301021

November 1992

\title{
Charge Screening, Large-N, and the Abelian Projection Model of Confinement
}

\author{
L. Del Debbio \\ Dipartimento di Fisica dell' Università, and I.N.F.N. \\ I-56100 Pisa, Italy \\ M. Faber" \\ Institut für Kernphysik \\ Wiedner Hauptstrasse 8-10 \\ A-1040 Wien, Austria \\ J. Greensite尸 \\ Physics and Astronomy Dept. \\ San Francisco State University \\ 1600 Holloway Ave. \\ San Francisco, CA 94132 USA
}

\footnotetext{
${ }^{1}$ Supported in part by the Fonds zur Förderung der wissenschaftlichen Forschung under Contract No. P7237-TEC.

${ }^{2}$ Supported by the U.S. Department of Energy under Grant No. DE-FG03-92ER40711.
} 


\begin{abstract}
We point out that the abelian projection theory of quark confinement is in conflict with certain large-N predictions. According to both large- $\mathrm{N}$ and lattice strongcoupling arguments, the perimeter law behavior of adjoint Wilson loops at large scales is due to charge-screening, and is suppressed relative to the area term by a factor of $1 / N^{2}$. In the abelian projection theory, however, the perimeter law is due to the fact that $N-1$ out of $N^{2}-1$ adjoint quark degrees of freedom are (abelian) neutral and unconfined; the suppression factor relative to the area law is thus only $1 / N$. We study numerically the behavior of Wilson loops and Polyakov lines with insertions of (abelian) charge projection operators, in maximal abelian gauge. It appears from our data that the forces between abelian charged, and abelian neutral adjoint quarks are not significantly different. We also show via the lattice strong-coupling expansion that, at least at strong couplings, QCD flux tubes attract one another, whereas vortices in type II superconductors repel.
\end{abstract}




\section{Introduction}

Large-N arguments are a useful way to critique some of the quark confinement mechanisms that have been proposed over the years. For example, the $Z_{N}$ fluxon mechanism [1] does not give a string tension for adjoint quarks at any length scale. This was shown to be in contradiction with large- $\mathrm{N}$ factorization, which predicts that: (i) the adjoint string tension $\sigma_{A}$ (from the confinement scale to the charge screening scale $L_{s}$ ) is roughly twice the fundamental string tension $\sigma_{F}$; and (ii) the distance $L_{s}$ where the adjoint flux tube breaks, due to charge screening, goes to $\infty$ as $N \rightarrow \infty$ [2]. Existing Monte Carlo calculations appear to be generally consistent with these large-N predictions [3]. A quite different mechanism is the "dual superconductor" idea, particularly as formulated by 't Hooft in abelian-projection gauges [4]; this formulation has been widely discussed in recent years [5-11]. It is obviously of interest to see whether the abelian projection idea is in agreement with large- $\mathrm{N}$ predictions.

At first sight, the abelian projection theory meets the large- $\mathrm{N}$ criteria quite well: adjoint quarks will indeed have a string tension $\sigma_{A} \approx 2 \sigma_{F}$ from the confinement scale to some intermediate distance, beyond which the adjoint quarks are unconfined [6]. However, as discussed in section 3, there is still a profound contradiction between large- $\mathrm{N}$ and abelian-projection predictions, namely, for an adjoint Wilson loop of area $\mathcal{A}$ and perimeter $\mathcal{P}$ we expect at large- $\mathrm{N}$ the leading behavior

$$
W(C)=N^{2}\left[e^{-\sigma \mathcal{A}-\mu \mathcal{P}}+\frac{1}{N^{2}} e^{-\mu^{\prime} \mathcal{P}}\right]
$$

while for abelian projection we find

$$
W(C)=N^{2}\left[e^{-\sigma \mathcal{A}-\mu \mathcal{P}}+\frac{1}{N} e^{-\mu^{\prime} \mathcal{P}}\right]
$$

This may not seem like a very serious difference - just a $1 / N$ vs. $1 / N^{2}$ suppression of the perimeter law term - but in fact it reveals something very fundamental about the abelian projection mechanism: In abelian projection gauge, certain adjoint quark colors are unconfined not because of charge screening, but rather because they are neutral with respect to the residual $U(1)^{N-1}$ gauge invariance, and this in turn leads to results that differ with large-N. The disagreement suggests certain numerical tests of the abelian projection idea, based on the behavior of Wilson loops and Polyakov lines with insertions of various abelian charge projection operators. It is found, in section 4 , that these projection operators do not make much difference so far as string tension and screening distance are concerned, and that the force between quarks which are 
neutral with respect to the abelian projection subgroup is about the same as the force between quarks which are charged in the abelian subgroup. These numerical results do not favor the abelian projection theory, and add to the negative body of evidence already presented in ref. [7, 8, 9]. We will comment on the apparently positive results presented in ref. 10, 11.

We will also comment, in the context of the lattice strong-coupling expansion, on the force between QCD flux tubes. Flux tubes in QCD have often been likened to Abrikosov vortices in type II superconductors. The force between vortices in type II superconductors is repulsive, due to the negative surface energy of the vortices, so it is natural to ask whether the force between QCD flux tubes is also repulsive. In the next section it will be shown that, at least at strong-couplings, the force between QCD flux tubes is actually attractive.

\section{Charge Screening and Flux Coalescence}

Strong-coupling calculations using the Kogut-Susskind Hamiltonian, or based on the Heat-Kernel action, as well as Monte-Carlo calculations in the scaling region with the Wilson action [3], all give the result that the force between a quark and an antiquark, with color charges in representation $R$ of the gauge group $S U(N)$, is initially proportional to the quadratic Casimir $C_{R}$ of the group representation. For any representation of $\mathrm{SU}(\mathrm{N})$ there is a generalization of the concept of triality in $\mathrm{SU}(3)$, known as the "N-ality" or, in the mathematical literature, the "class" of the representation. Another result of strong-coupling [2] and Monte-Carlo [3] calculations is that at some distance beyond the confinement scale, at a screening distance denoted $L_{s}$, the force between quarks drops abruptly. Beyond the screening distance, the force becomes proportional to the smallest Casimir $C_{L}$ among all representations with the same N-ality as representation $R$. This is known as "charge-screening", and it has a simple physical explanation: As the quark-antiquark pair separates and and the energy stored in the flux-tube increases, it becomes energetically favorable to paircreate gluons. These gluons bind to the quark and the antiquark and, although gluons cannot change the $\mathrm{N}$-ality of the quark charge, they can lower the effective charge of the quark-gluon state to a representation $L$ of the same $\mathrm{N}$-ality, with the lowest possible Casimir $C_{L}$. A particular example is the case of having a pair of quarks in the adjoint representation. The adjoint representation has $\mathrm{N}$-ality $=0$, the same as a singlet. A pair of gluons, binding to each quark, can reduce the charge to a singlet, 
and therefore just break the flux-tube between the adjoint quarks. The mechanism is exactly the same as in QCD with dynamical quarks, where a flux-tube is broken by quark-antiquark production. For this reason, in pure QCD, we expect an adjoint Wilson loop to have an area law from the confinement scale up to a distance $L_{s}$, after which it is screened to perimeter law. This is indeed what is seen in numerical experiments [3].

An important point, especially for the purposes of this article, is that diagramatically the charge-screening process is always suppressed by a factor of $1 / N^{2}$ in pure QCD; this is true whether the process is represented by lattice strong-coupling diagrams, as shown in Fig. 1, or by high-order Feynman diagrams. Feynman diagrams can be classified according to their associated powers of $1 / N^{2}$, and diagrams of leading order - the planar diagrams - satisfy the factorization property

$$
<(\operatorname{Tr} A)(\operatorname{Tr} B)>=<\operatorname{Tr} A><\operatorname{Tr} B>
$$

For this reason $\sigma_{A}=2 \sigma_{F}$ in the large- $\mathrm{N}$ limit; it is also the reason why charge screening must be a non-planar process. Non-planar diagrams, whether Feynman or lattice strong-coupling, are suppressed (in pure QCD) by a factor of at least $1 / N^{2}$ relative to the planar diagrams. Therefore, we conclude on these very general grounds that for an adjoint Wilson loop

$$
W_{A}(C)=N^{2}\left[\exp \left[-2 \sigma_{F} \mathcal{A}-\mu \mathcal{P}\right]+\frac{1}{N^{2}} \exp \left[-\mu^{\prime} \mathcal{P}\right]\right]
$$

In strong-coupling one can easily calculate where the perimeter behavior of the second term takes over from the area behavior; for square $L \times L$ loops this occurs for

$$
L>\left[\frac{\ln N}{\sigma_{F}}\right]^{1 / 2}
$$

which diverges as $N \rightarrow \infty$.

An effect which is very closely related to charge screening is the phenomenon of flux coalescence. This effect is relevant to the question of whether the force between QCD flux tubes is repulsive, as it is for Abrikosov vortices in type II superconductors, or attractive.

Let us consider two quark-antiquark pairs with the axes through each pair parallel; these are represented by the two parallel $R \times T$ Wilson loops shown in Fig. 2, which are separated by a distance $L>>R$, and which have the same orientation. Denote the expectation value of the product of these loops by $D(R, T, L)$. Then the interaction 
energy between the loops, which represents the interaction of the flux tubes between the quark-antiquark pairs, is just

$$
V(L)=-\lim _{T \rightarrow \infty} \frac{\partial}{\partial T} \ln \left[\frac{D(R, T, L)}{D(R, T, \infty)}\right]
$$

and

$$
D(R, T, L)=D(R, T, \infty)+R T(L+1)\left(A_{a}-A_{b}\right)
$$

where, to leading order

$$
D(R, T, \infty)=N^{2} e^{-2 \sigma_{F} R T}
$$

The leading strong-coupling contributions to $A_{a}$ and $A_{b}$ are indicated schematically in Fig. 3.

We will calculate $V(L)$ using the heat-kernel lattice action

$$
\begin{aligned}
e^{S} & =\prod_{p} e^{S_{p}} \\
e^{S_{p}} & =\sum_{r} d_{r} \chi_{r}\left(U_{p}\right) \exp \left[\frac{-C_{r}}{N \beta}\right]
\end{aligned}
$$

where the product extends over all oriented plaquettes $p$ and the summation runs over all inequivalent irreducible representations $r$ of dimension $d_{r} \cdot \chi_{r}\left(U_{p}\right)$ is the character of the group element $U_{p} \in S U(N)$ in the representation $r$, and $C_{r}$ is the eigenvalue of the quadratic Casimir operator in this representation. For quarks in the fundamental (defining) representation, the plaquette with character $\chi_{r}^{\dagger}\left(U_{p}\right)$ in the middle of the tube in Fig. 2a can only be in the symmetric $(r=s)$ or antisymmetric $(r=a)$ representations formed from the product of two fundamental defining representations. The interaction potential is then found to be

$$
V(L)=-(L+1) e^{-4 \sigma_{F} L} R\left[\frac{N-1}{2 N} \exp \left[\frac{1+N}{N^{2} \beta}\right]+\frac{N+1}{2 N} \exp \left[\frac{1-N}{N^{2} \beta}\right]-1\right]
$$

Expanding the exponents in a Taylor series, $V(L)$ to leading order in $1 / N$ is given by

$$
V(L)=-\frac{1}{2 \beta^{2}} \frac{1}{N^{2}} R(L+1) e^{-4 \sigma_{F} L}
$$


which is an attractive potential at large $L>R$. Flux tubes therefore tend to attract one another, by a process which can be viewed as glueball exchange.

For separations $L<<R$ flux tubes do not only attract, they will actually tend to coalesce into a single flux tube. In this case

$$
D(R, T, L)=D(R, T, \infty)+D_{c}(R, T, L)
$$

where the leading contribution to $D_{c}$ is shown in Fig. 4. In this figure there is a single sheet of plaquettes, of area $R \times T$, in a fundamental representation of N-ality $\mathrm{N}-2$. For $R<<N L$ the disconnected diagrams dominate, and the energy of the system is simply the sum of the two disconnected flux tubes

$$
E_{0}=2 \sigma_{F} R=\frac{N^{2}-1}{N^{2} \beta} R
$$

whereas, if $R>>N L$, it is energetically favorable for the two flux tubes to coalesce, as represented in Fig. 4, and the energy is

$$
E_{c}=\sigma_{a} R+2 \sigma_{F} L=\frac{(N-2)(N+1)}{N^{2} \beta} R+\frac{N^{2}-1}{N^{2} \beta} L
$$

It is clear that $E_{c}<E_{0}$, i.e. coalescence is favored, for $R>N L$.

The calculation above can be easily generalized to the case of $n$ quark-antiquark pairs, with axes nearby and aligned parallel to one another. Once again, for quark separations $L$ much less than quark-antiquark separations $R$, it is energetically favorable for the $n$ flux tubes between each quark-antiquark pair to coalesce into a single tube, as shown in Fig. 5. Its string tension is determined by the eigenvalue of the quadratic Casimir operator of the lowest dimensional representation $r$ of N-ality $(N-n) \bmod N$, which is again a fundamental representation of dimension $d_{r}=\left(\begin{array}{l}N \\ n\end{array}\right)$. It is clear that coalescence may result in considerable lowering of the energy of the system, compared to the configuration of $n$ separate flux tubes between each $q \bar{q}$ pair; the ratio between the energies of these two different configurations being, for $R>>L$,

$$
r=\frac{\sigma_{L}}{n \sigma_{F}}=\frac{C_{L}}{n C_{F}}=1-\frac{n-1}{N-1}
$$

For $\mathrm{n}=2$ in $\mathrm{SU}(3)$, this ratio is just $r=\frac{1}{2}$.

The phenomenon of flux-tube coalesence implies that the force between nearby QCD flux tubes is attractive, at least at strong couplings, in contrast to the situation

\footnotetext{
${ }^{3}$ In fact, this potential is also attractive at small $L$, given $\sigma_{F}=C_{F} /(N \beta)>1$, which is the case at strong-couplings. But at $L<<R$, flux tube coalescence, discussed below, is the dominant effect.
} 
in type-II superconductivity. If strong-coupling calculations are any guide, and if confinement in QCD is analogous to (dual) superconductivity, then the analogy would presumably be to type I superconductivity. More importantly, both color charge screening and the attractive force between flux tubes are due to non-planar, $1 / N^{2}$ suppressed processes. We will now consider whether the abelian projection theory is consistent with this suppression factor.

\section{The Abelian Projection Theory}

The abelian projection theory of quark confinement was put forward in ref. [4]. In this theory a gauge-fixing condition is first chosen to break the $\mathrm{SU}(\mathrm{N})$ symmetry down to the Cartan subgroup $U(1)^{N-1}$. Monopoles are then identified with singularities in the gauge-fixing condition, and condensation of these monopoles is invoked to explain confinement of particles charged with respect to the $U(1)^{N-1}$ subgroup. The confinement mechanism is analogous that of compact QED, with gluons associated with the Cartan subalgebra playing the role of the photon field which forms the flux tube. These "diagonal" gluons are uncharged with respect to the residual $U(1)^{N-1}$ symmetry; all other gluons are charged with respect to the residual symmetry, and, like quarks, are confined by flux tubes.

Consider the force between two quarks with charges in adjoint representation, in the abelian projection theory. For simplicity (and because it makes little difference in the large- $\mathrm{N}$ limit), take the group to be $U(N)$ rather than $S U(N)$, so the abelian subgroup is $U(1)^{N}$. Let $g_{i}$ denote an abelian charge of magnitude $g$ in the i-th $U(1)$ subgroup. A quark in the fundamental (defining) representation has $N$ color degrees of freedom, and from the abelian-projection point of view, each of these degrees of freedom corresponds to a quark $Q_{F}^{i}$ with a different abelian charge $g_{i}, 1 \leq i \leq N$. Likewise, the $N^{2}$ degrees of freedom of a quark in the adjoint representation can be be grouped according to abelian charge: there are $N(N-1)$ quarks $Q_{A}^{i j}, i \neq j$ with charge $\left(g_{i},-g_{j}\right)$, and there are $N$ quarks $Q_{A}^{i i}$ which are neutral with respect to the $U(1)^{N}$ subgroup.

Since the flux-tube between static adjoint quarks is neutral with respect to the abelian subgroup in the abelian projection picture (flux tubes are formed from the "photon" fields), the charges of the quarks themselves must be correllated in order to have a neutral composite state. The quark-content of the composite state, in an abelian-projection gauge, must then be 


$$
\operatorname{Tr} Q\left[x_{1}\right] Q\left[x_{2}\right]=\sum_{i \neq j} Q^{i j}\left[x_{1}\right] Q^{j i}\left[x_{2}\right]+\sum_{i} Q^{i i}\left[x_{1}\right] Q^{i i}\left[x_{2}\right]
$$

Consider the $N(N-1)$ contributions to the first sum. Each contribution represents two quarks with equal and opposite charges $\left(g_{i},-g_{j}\right)$ and $\left(-g_{i}, g_{j}\right)$ respectively. Since each quark is charged in two different abelian subgroups, there will be two flux tubes between them, each with string tension $\sigma_{F}$. Thus the net string tension for these quarks is $\sigma_{A}=2 \sigma_{F}$, in agreement with large-N. The $N$ quarks of the second sum, however, are neutral with respect to the confining abelian $U(1)^{N}$ subgroup; these neutral quarks are unconfined. For an adjoint Wilson loop of area $\mathcal{A}$ and perimeter $\mathcal{P}$, we therefore expect

$$
W(C)=N(N-1) \exp \left[-2 \sigma_{F} \mathcal{A}-\mu P\right]+N \exp [-\mu \mathcal{P}]
$$

for the following reason: Given that the abelian "charged" gluons are confined and the flux tube is neutral, the quarks cannot exchange charge between them, and a quark can only change its abelian charge by emission and reabsorbtion of a charged gluon. Even allowing for such virtual processes, the abelian charge of each quark + gluon-cloud cannot change. Thus the color sum in a Wilson loop can be expressed as a sum of loops, each representing a particular $\left(g_{i},-g_{j}\right)$ charge running around the loop. The $N(N-1)$ charged adjoint quarks $(i \neq j)$ contribute to the first term in (17), and the $N$ neutral, unconfined quarks contribute to the second term.

The expression (17) above for the adjoint Wilson loop is in disagreement with the large-N prediction. In the large- $\mathrm{N}$ analysis, all quark colors are on the same footing, and the perimeter term comes about through charge-screening, and not because any subset of quark charges is oblivious to the confining force. More importantly, according to large- $\mathrm{N}$, the coefficient of the perimeter term is $O(1)$, rather than $O(N)$ as in the abelian projection theory.

To make the same point in a slightly different way, consider the trace of an adjoint Wilson loop in $S U(N)$

$$
\begin{aligned}
\operatorname{Tr}_{A}\left[P \exp \left[i \oint A^{a} L_{a}\right]\right] & =\sum_{m} \operatorname{Tr}_{A}[\mid m)\left(m \mid P \exp \left[i \oint A^{a} L_{a}\right]\right] \\
& =\sum_{m}\left(m\left|P \exp \left[i \oint A^{a} L_{a}\right]\right| m\right)
\end{aligned}
$$

where $L_{a}$ are the group generators and sum is over all members $\mid m$ ) of the multiplet. The confining force can only come from coupling of the adjoint quarks to the gluons 
in the Cartan subalgebra, with generators denoted $H_{i}(i=1, \ldots, N)$. Therefore, in an abelian projection gauge such as the maximal abelian gauge, following the reasoning of ref. [12],

$$
\begin{aligned}
<\operatorname{Tr}_{A}\left[P \exp \left[i \oint A^{a} L_{a}\right]\right]> & \approx<\operatorname{Tr}_{A}\left[\exp \left[i \oint A^{i} H_{i}\right]\right]> \\
& =\sum_{m=1}^{N^{2}-1}<\left(m\left|\exp \left[i \oint A^{i} H_{i}\right]\right| m\right)> \\
& =\sum_{m=1}^{N^{2}-1}<\exp \left[i \oint A^{i} \lambda_{i}^{(m)}\right]>
\end{aligned}
$$

where the $\lambda_{i}^{(m)}$ are eigenvalues

$$
\left.\left.H_{i} \mid m\right)=\lambda_{i}^{(m)} \mid m\right)
$$

Then, making use of the fact that the multiplicity of zero-weight (all $\lambda_{i}=0$ ) states in the adjoint representation is $N-1$, we have

$$
W(C) \approx \sum_{\substack{m=1 \\ \text { (non-zero } \\ \text { weight states) }}}^{N(N-1)} e^{-\sigma^{(m)} \mathcal{A}-\mu^{\prime} \mathcal{P}}+(N-1)
$$

Ignoring the coupling of adjoint quarks to gluons which are not in the Cartan subalgebra, which was the approximation made in eq. (19), has the effect of dropping selfenergy (perimeter law) contributions to the zero-weight and underestimating them for the non-zero weight states. If these contributions are included perturbatively, the final answer would be

$$
W(C) \approx \sum_{\substack{m=1 \\ \text { (non-zero } \\ \text { weight states) }}}^{N(N-1)} e^{-\sigma^{(m)} \mathcal{A}-\mu \mathcal{P}}+(N-1) e^{-\mu \mathcal{P}}
$$

Again, the deconfined term is $O(N)$, rather than $O(1)$ as expected by large-N arguments. 


\section{Numerical Tests}

The fact that the abelian projection theory disagrees with large- $\mathrm{N}$ predictions should not be too surprising. As noted above, the deconfinement of adjoint loops according to the abelian projection is simply due to the fact that $N-1$ out of $N^{2}-1$ adjoint quarks are neutral with respect to the $U(1)^{N-1}$ subgroup, and are therefore unconfined in the abelian projection theory. This gives a suppression factor of only $1 / N$ to the unconfined contribution. Charge-screening, which involves pair-creating gluons that bind to the adjoint quarks, is a completely different mechanism, and just gives the usual non-planar suppression factor of $1 / N^{2}$. This disagreement doesn't necessarily mean that the abelian projection theory is wrong; it could be the large- $\mathrm{N}$ arguments that have somehow gone astray. But such a clear difference in the two approaches does suggest a simple numerical test.

Let us consider the case of $N=2$, with $H=L_{3}$ to be the generator of the Cartan-subalgebra. Then eq. (19) becomes

$$
\begin{aligned}
W(C) & \approx \sum_{m=-1}^{1}<\exp \left[i m \oint A^{3}\right]> \\
& =2 e^{\sigma^{(1)} \mathcal{A}}+1
\end{aligned}
$$

or, correcting for the self-energy (perimeter) contributions

$$
W(C)=2 e^{\sigma^{(1)} \mathcal{A}-\mu \mathcal{P}}+e^{-\mu \mathcal{P}}
$$

In other words, the abelian-projection prediction is that, in an abelian projection gauge

$$
\begin{aligned}
<(1|P \exp [i \oint A]| 1)> & =<(-1|P \exp [i \oint A]|-1)>=e^{-\sigma^{(1)} \mathcal{A}-\mu \mathcal{P}} \\
<(0|P \exp [i \oint A]| 0)> & =e^{-\mu \mathcal{P}}
\end{aligned}
$$

The inner product $<(0|\ldots| 0)>$ can be thought of as creation of a quark-antiquark pair with zero abelian charge, which run around the loop. The only way to change the abelian charge of the pair would be for the quarks to exchange a gluon with non-zero abelian charge. However, this process should be suppressed at the confinement scale, since the charged gluons are presumably confined, and therefore only contribute to the self-energy of each quark. The string tension of the $\langle(0|.| 0)>$. term should 
therefore be zero in an abelian-projection gauge; there should be no area suppression whatever, in any range of quark separations. This is an easy prediction to test.

Let $U(C)$ represent a product of link variables along the path $C$. In $S U(2)$ this can always be expressed as

$$
U(C)=a_{0} \mathbf{1}+i a_{k} \sigma^{k}
$$

where the $\sigma^{k}$ are the Pauli matrices. Then, in the adjoint representation

$$
\begin{aligned}
U_{++}(C) \equiv\left(1\left|U_{A}(C)\right| 1\right) & =a_{0}^{2}-a_{3}^{2}+2 i a_{0} a_{3} \\
U_{--}(C) \equiv\left(-1\left|U_{A}(C)\right|-1\right) & =a_{0}^{2}-a_{3}^{2}-2 i a_{0} a_{3} \\
U_{00}(C) \equiv\left(0\left|U_{A}(C)\right| 0\right) & =2\left(a_{0}^{2}+a_{3}^{2}\right)-1
\end{aligned}
$$

Define the charged and neutral Wilson loops

$$
\begin{aligned}
& W_{c}(C)=<U_{++}(C)>=<U_{--}(C)> \\
& W_{0}(C)=<U_{00}(C)>
\end{aligned}
$$

together with the corresponding Creutz ratios $\chi_{c}[R, T]$ and $\chi_{0}[R, T]$. As discussed above, the abelian projection prediction is that $\chi_{0}[R, T]=0$ (or, at least, that $\left.\chi_{0}<<\chi_{c}\right)$ in an abelian projection gauge.

We have computed these Creutz ratios by lattice Monte Carlo, in $\mathrm{D}=3$ dimensions with a Wilson action at $\beta=5$, which is just inside the $\mathrm{D}=3$ scaling region. The charged and neutral loops were evaluated in maximal abelian gauge. The results obtained after 153000 update iterations, for $\chi_{0}[R, R], \chi_{c}[R, R]$, and for $\chi[R, R]$ (the Creutz ratio of gauge-invariant adjoint $j=1$ loops), are shown in Fig. 6. Creutz ratios for the fundamental $\left(j=\frac{1}{2}\right)$ loop $\chi_{F}(R, R)$ are also displayed in Fig. 6. It can be seen that Creutz ratios in the $j=\frac{1}{2}$ and $j=1$ representations are in the proportion predicted by large-N, which is a ratio of Casimirs

$$
\frac{\chi[R, R]}{\chi_{F}[R, R]} \approx \frac{8}{3}
$$

It can also be seen that $\chi_{0}$ is not zero, and shows no tendency to go zero faster than $\chi_{c}$. In fact, although $\chi_{0}$ is smaller than $\chi_{c}$, the difference is only about $10 \%$; this difference does not appear to grow with loop size. Thus, while there may perhaps be 
some effect attributable to abelian monopoles, the effect seems rather small; certainly it is not sufficient to explain confinement in this region. This result seems entirely consistent with the results in ref. [8].

We have also studied the correlation of Polyakov lines in $\mathrm{D}=4$ dimensions and maximal abelian gauge

$$
\begin{aligned}
P_{0}(R) & =<U_{00}\left[L_{1}\right] U_{00}\left[L_{2}\right]> \\
P_{c}(R) & =<U_{++}\left[L_{1}\right] U_{--}\left[L_{2}\right]>
\end{aligned}
$$

where $L_{1,2}$ are parallel Polyakov loops, 2 lattice spacings in length, separated by $\mathrm{R}$ lattice spacings in the spatial hyperplane. Because of the small time extension, we must work at a strong coupling - in our case $\beta=1.8$ - to avoid the deconfinement transition. Although this is at strong-coupling, it is at least close to the strongto-weak coupling transition point, and we can see if there is any tendency for the "neutral" correllations $P_{0}(R)$ to behave differently from the "charged" correlations $P_{c}(R)$. The data is shown in Fig. 7. Charge screening sets in at about 2 lattice spacings, and there does not appear to be any difference in the behavior of $P_{0}$ and $P_{c}$.

For the numerical simulations, we used an heat-bath algorithm, running on a $16^{3} \times 2$ lattice at $\beta=1.80$. The results were obtained averaging over 1000 configurations, after 20000 sweeps of thermalization. The continous lines in Fig. 7 are the results of fit for the thermodynamical mixing of two interaction channels as described in ref. [13]. For the first Boltzmann factor we used the lattice version of a Coulomb plus linear potential plus self energy and via the second term we took the screening of charges into account.

The conclusion of this numerical work is that there seems to be no appreciable difference in the forces between abelian charged and abelian neutral adjoint quarks, at the couplings and separations we have investigated. This evidence does not favor the abelian projection theory.

It may be appropriate, at this point, to comment on certain other Monte Carlo investigations of the abelian projection theory. Numerical work on this problem was initiated by Kronfeld et. al [5], who found a drop in the monopole density at the deconfinement phase transition, in "maximal abelian gauge" defined as the gauge which maximizes the quantity

$$
Q=\sum_{x} \sum_{\mu=1}^{4} \operatorname{Tr}\left[\sigma_{3} U_{\mu}(x) \sigma_{3} U_{\mu}^{\dagger}(x)\right]
$$


More recent investigations by Del Debbio et. al. have shown, however, that the definition of monopole density is plagued by lattice artifacts, and is not at all a good order parameter for confinement [7]. For example, it is found the monopole density neither shows correllation with the string tension with cooling, nor a drop across the deconfinement phase transition. These problems may be alleviated by a more appropriate definition of the monopole creation operator, but we will not pursue that issue here.

An alternate line of investigation is to see if the electric flux inside the flux tube is dominated by the Cartan subalgebra; e.g. in $\mathrm{SU}(2)$, with the choice of maximal abelian gauge above, one checks to see if the field-strength is proportional to $\sigma_{3}$. Let $U_{\mu \nu}$ be the plaquette variable. One defines the field strength on the lattice as $\left.F_{\mu \nu}^{a}=\operatorname{Tr}\left[U_{\mu \nu}-U_{\mu \nu}^{\dagger}\right] \sigma^{a}\right] / 4 i$, as well as the quantities

$$
J^{a}=\frac{<D^{a} \operatorname{Tr} W>}{<\operatorname{Tr} W>}-<D^{a}>
$$

where $W$ is a Wilson loop in the $\mu \nu$ plane; and $D^{a}=\sum\left(F_{\mu \nu}^{a}(x)\right)^{2} / n_{P}$, where the sum is over central plaquettes in the minimal area bounded by the loop, furthest from the boundary. $n_{P}$ is the number of central plaquettes. Let $J=J^{1}+J^{2}+J^{3}$, and define the ratio

$$
\rho=\frac{J^{3}}{J}
$$

If $\rho \approx 1$ this would tend to confirm the abelian projection theory, while $\rho \approx 1 / 3$ would tend to refute it. The result found in [8, 9] was $\rho \approx 1 / 3$.

On the other hand, Hioki et. al. [10] have argued that this ratio should be defined using abelian loops in the numerator. An abelian link is defined as the diagonal part of the link variable, rescaled to restore unitarity. An abelian loop is a loop constructed from abelian links. Let $u_{\mu \nu}$ be a $1 \times 1$ abelian loop, and define

$$
\begin{aligned}
\rho_{A} & =\frac{J_{A}}{J} \\
J_{A} & =\frac{<\frac{1}{n_{P}} \sum f_{\mu \nu}^{2} \operatorname{Tr} W>}{<\operatorname{Tr} W>}-<f_{\mu \nu}^{2}> \\
f_{\mu \nu} & =\left(u_{\mu \nu}-u_{\mu \nu}^{*}\right) / 2 i
\end{aligned}
$$

With these definitions, Hioki et. al. find $\rho_{A}$ actually greater than 1 (up to $\rho_{A} \approx 1.6$, in fact). 
We regard conclusions based on the enhancement of abelian loops, both in ref. 10] and also in [11] as very misleading for the following reason: Maximal abelian gauge simply makes links as diagonal as possible; the enhancement of abelian loops (termed "abelian dominance") which was found in [11] is a simple consequence of this fact. It is therefore crucial to choose observables whose behavior will really test the abelian projection theory, rather than just display this particular aspect of the gauge condition. The behavior of abelian Wilson loops, and the $\rho_{A}$ quantity defined above, do not meet that criterion.

The following calculation will illustrate the point. Instead of gauge-fixing to maximal abelian gauge, let us fix to another, "x-y" maximal abelian gauge, introduced in ref. [8], which is defined to maximize the quantity

$$
Q=\sum_{x} \sum_{\mu=1}^{2} \operatorname{Tr}\left[\sigma_{3} U_{\mu}(x) \sigma_{3} U_{\mu}^{\dagger}(x)\right]
$$

This gauge forces links in the $\mathrm{x}$ and $\mathrm{y}$ directions only to be as diagonal as possible. Since there is no requirement, in the abelian projection theory, that the gauge-fixing condition must be spherically symmetric, this gauge should be as good as the usual maximal abelian gauge. We now compute $\rho_{A}$ by Monte Carlo separately for loops oriented in the $\mathrm{x}-\mathrm{y}$, and $\mathrm{z}$-t planes. The results have only been computed for rather small loops, but we feel they already show how things go. The loops are from $\mathrm{R}=1$ to $\mathrm{R}=4$ lattice spacings wide, and $\mathrm{T}=4$ lattice spacings high. Half of the plaquettes in the minimal area of the loop were used for calculating $J_{A}$; these are the $2 \times R$ plaquettes which are one lattice spacing away from loop boundaries in the $T$ direction.

The results of this calculation, performed in $\mathrm{D}=4$ dimensions at $\beta=2.4$, are shown in Fig. 8. If one accepts the proposition that $\rho_{A} \approx 1$ is evidence for the abelian projection theory, then it would seem from this data that monopoles are responsible for confinement in the $\mathrm{x}-\mathrm{y}$ plane, but not in the z-t plane. That conclusion, we feel, is nonsense. A much more reasonable explanation is that, because links are nearly diagonal in the $\mathrm{x}-\mathrm{y}$ plane, loops which are built from the diagonal part of the links pretty well approximate the full Wilson loop. Conversely, in the z-t plane, the results look much as if there were no gauge-fixing at all.

As an additional check, we have also computed $\rho_{A}$ for a small $R=2, T=4$ loop at $\beta=2.8$, in ordinary maximal abelian gauge. At this value of $\beta$, for such a small loop, there should be no flux tube formed; yet we find $\rho_{A}=0.88$. This is another indication that the large value of $\rho_{A}$ is a gauge effect, which has nothing to do with 
flux tube formation.

This calculation illustrates the fact that, in maximal abelian gauge, one must ensure that the observables chosen are relatively insensitive to the diagonality of links which is enforced by the gauge. This is the case for the $J^{a}$ observables of eq. (32), whose sum is directly related to the energy density in the flux tube; it is not the case for the $J_{A}$ observable of eq. (34), which is not related directly to the energy density.

\section{Conclusions}

A prediction of the abelian projection theory of confinement is that, in an abelian projection gauge, adjoint quark colors which are neutral with respect to the remnant $U(1)^{N-1}$ symmetry are oblivious to the confining force. This prediction turns out to conflict with large-N arguments, and can be tested by looking for the absence of an area-law term, over any length scale, in Wilson loops and Polyakov lines with appropriate insertions of abelian-neutral projection operators. We have found, instead, that insertion of abelian neutral (and abelian charged) projection operators has very little effect on the value of the string tension extracted from the loop. Assuming that the QCD flux tube in abelian projection gauge is abelian neutral (since it is supposed to be formed by the "photon" fields), this means that there is no significant difference in the forces between abelian neutral adjoint quarks, and between abelian charged adjoint quarks. Thus our data is consistent with large- $\mathrm{N}$ expectations, and in conflict with the abelian-projection theory, which holds that the confining force is sensitive mainly to the $U(1)^{N-1}$ charge.

We have also examined the claim of "abelian dominance" in maximal abelian gauge, found in [10, 11]. We believe that our data in an "x-y maximal abelian gauge" (together with previous work along these lines in ref. [8]), demonstrates that this enhancement of abelian loops is purely a gauge effect, with no relevance at all to the physics of confinement.

Finally, we have calculated the force between QCD flux tubes in the lattice strongcoupling expansion, and find that this force is attractive. If the strong-coupling result survives in the continuum theory (and there is reason, based on the general notion of flux coalescence, to think that it might), then QCD flux tubes are not analogous to Abrikosov vortices in type II superconductors, which tend to repel one another.

In general, theories of confinement which rely on analogies to abelian gauge theo- 
ries will tend to identify a small subset of the degrees of freedom, e.g. associated with the $Z_{N}$ center or the Cartan subgroup of the full gauge group, as being especially important for quark confinement. Such a subset becomes negligibly small compared to the total number of degrees of freedom in the $N \rightarrow \infty$ limit, and it is therefore not surprising that such theories will somewhere contradict results based on large- $\mathrm{N}$ counting arguments. One theory of confinement which is based rather explicitly on the large-N picture is the "gluon-chain" model of flux-tube formation, advocated by one of us in ref. [14]. This model is consistent with all large-N predictions, and also has some numerical support [15]. It is relevant here as an example of a confinement mechanism in non-abelian gauge theories which has no abelian counterpart; we believe that this must be true of any mechanism which is consistent with large- $\mathrm{N}$ results.

\section{Acknowlegments}

We thank the organizers of the Hadron Structure 91 meeting held in Stara Lesna, Czechoslovakia, where this work was begun. We also thank J. Hošek for drawing our attention to the relevance of vortex repulsion to dual-superconductor models. M.F. is grateful to R. Dirl and P. Kasperkovitz for valuable suggestions concerning group-theoretical aspects of this investigation; J.G. acknowledges the hospitality of the Niels Bohr Institute and the Lawrence Berkeley Laboratory. The computer calculations were carried out in Tallahassee, Florida and Pisa, Italy; the Florida portion was supported by the Florida State University Supercomputer Computations Research Institute, which is partially funded by the U.S. Department of Energy through Contract No. DE-FC05-85ER250000.

\section{References}

[1] G. 't Hooft, Nucl. Phys. B138 (1978) 1; G. Mack, in "Recent Developments in Gauge Theories," Proceedings of the 1979 Cargese Summer Institute, ed. G 't Hooft et. al. (Plenum, New York, 1980).

[2] J. Greensite and M. Halpern, Phys. Rev. D27 (1983) 2545.

[3] M. Faber, W. Kleinert, M. Müller, and S. Olejnik, in "Hadron Structure 91", Proceedings of the HS91 Conference in Stara Lesna, Czechoslovakia, (Slovak 
Acad. of Sci., Bratislava,1991); H. Faber and H. Markum, Nucl. Phys. B (Proc. Suppl.) 4 (1988), 204; C. Michael, Nucl. Phys. B259 (1985) 58; J. Ambjorn, P. Olesen, and C. Peterson, Nucl. Phys. B240 [FS12] (1984) 533.

[4] G. 't Hooft, Nucl. Phys. B190 [FS3] (1981) 455.

[5] A. Kronfeld, M. Laursen, G. Schierholz, and U.-J. Wiese, Nucl. Phys. B293 (1987) 461;

[6] C. Rosenzweig, Phys. Rev. D38 (1988) 1934.

[7] L. Del Debbio, A. Di Giacomo, M. Maggiore, and S. Olejnik, Phys. Lett. B267 (1991) 254.

[8] J. Greensite and J. Iwasaki, Phys. Lett. B255 (1991) 415; J. Greensite and J. Winchester, Phys. Rev. D40 (1989) 4167.

[9] A. Di Giacomo, M. Maggiore, and S. Olejnik, Nucl. Phys. B347 (1990) 441.

[10] S. Hioki, S. Kitahara, S. Kiura, Y. Matsubara, O. Miyamura, S. Ohno and T. Suzuki, Phys. Lett. B272 (1991) 326.

[11] T. Suzuki and I. Yotsuyanagi, Phys. Rev. D42 (1990) 4257.

[12] J. Smit and A. van der Sijs, Nucl. Phys. B355 (1991) 603.

[13] M. Müller, W. Beirl, M. Faber, H. Markum, Nucl. Phys. B (Proc.Suppl.) 26 (1992) 423.

[14] J. Greensite, Nucl. Phys. B249 (1985) 263.

[15] J. Greensite, Nucl. Phys. B315 (1989) 663. 


\section{Figure Captions}

Fig. 1 Leading strong-coupling contributions to the adoint Wilson loop. (a) the $O\left(N^{2}\right)$ area contribution; (b) the $O(1)$ perimeter contribution.

Fig. 2 Two parallel quark-antiquark flux tubes realized by two parallel Wilson loops of the same orientation.

Fig.3 Diagrams used in computing flux-tube interactions (eq. (7)): (a) term $A_{a}$, (a plaquette in the middle of a tube); (b) term $A_{b}$. It is necessary to sum over the position of the tube in the sheet, and over the position of the "middle" plaquette along the tube.

Fig. 4 Leading contribution for parallel Wilson loops at $L<<R$. The sheet in the middle (bounded by the heavy solid line) is in the fundamental representation of N-ality $N-2$.

Fig. 5 Coalescence of $n$ quark-antiquark flux tubes into a single flux tube of N-ality $(N-n) \bmod N$.

Fig. 6 Creutz ratios of adjoint $(j=1)$ Wilson loops. Stars are the usual $\chi$ ratios, diamonds are ratios $\chi_{0}$ of loops with abelian-neutral $(m=0)$ projection operators; vertical crosses are ratios $\chi_{c}$ of loops with abelian-charged $(m= \pm 1)$ projection operators, evaluated in maximal abelian gauge. The errors on loops at $R=1,2,3 a$ are negligible, errors at $R=4 a$ are about $\pm 10 \%$. Also shown (by diagonal crosses) is $\chi_{F}$, the usual Creutz ratio in the fundamental $\left(j=\frac{1}{2}\right)$ representation. Simulation is in $\mathrm{D}=3$ dimensions at $\beta=5$.

Fig. 7 The potential $V(R)$ extracted from Polyakov lines of extension $N_{t}=2$ lattice spacings. The potentials for "neutral" (diamonds) and "charged" (vertical crosses) sources in maximal abelian gauge of $\mathrm{SU}(2)$ are compared with the ungauged potentials (stars) in the adjoint representation. For comparison we show also the potential between doublet sources. The statistical errors start to get larger than the symbols in the screening region and can be estimated from the fluctuations with the distance $\mathrm{R}$. The full lines are the results of fits with a sum of two Boltzmann factors. 
Fig. $8 \rho_{A}$ in the "x-y maximal abelian" gauge. Crosses represent $\rho_{A}$ extracted from loops in the $\mathrm{x}-\mathrm{y}$ plane; diamonds represent $\rho_{A}$ extracted from loops in the z-t plane. Squares are values of $\rho_{A}$ taken with no gauge fixing. 

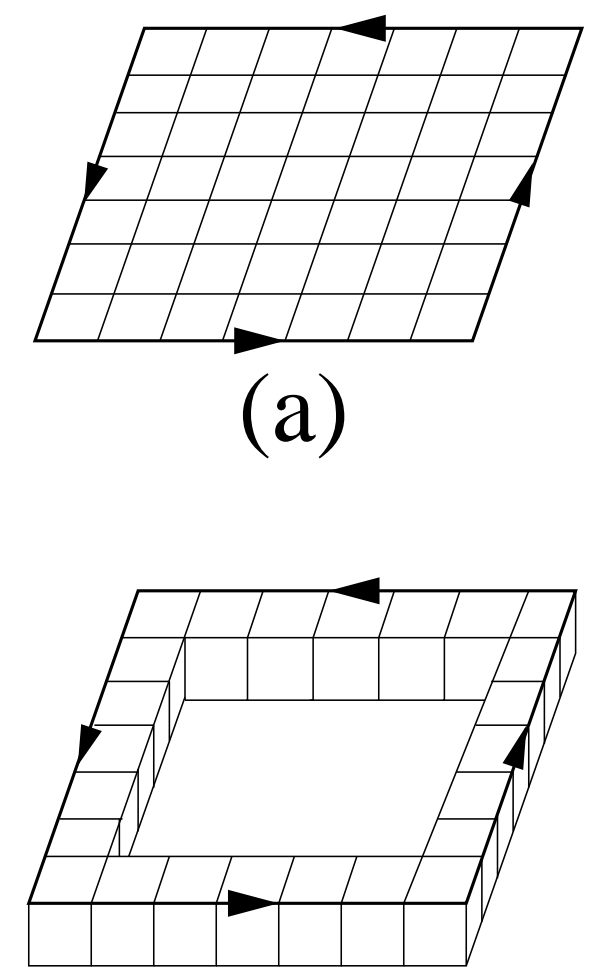

(b)

Figure 1 


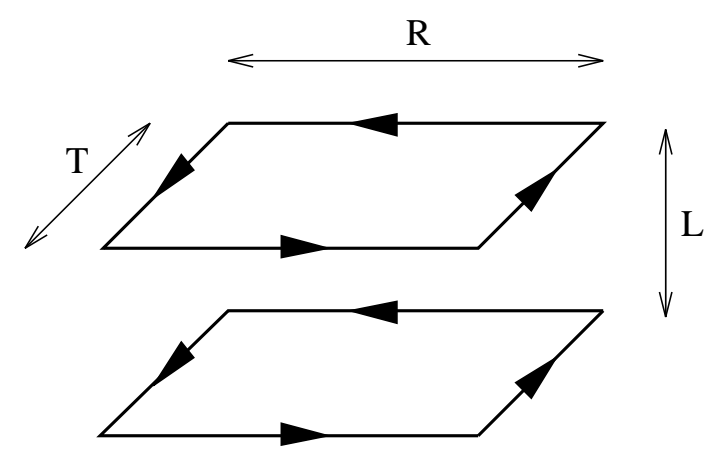

Figure 2 

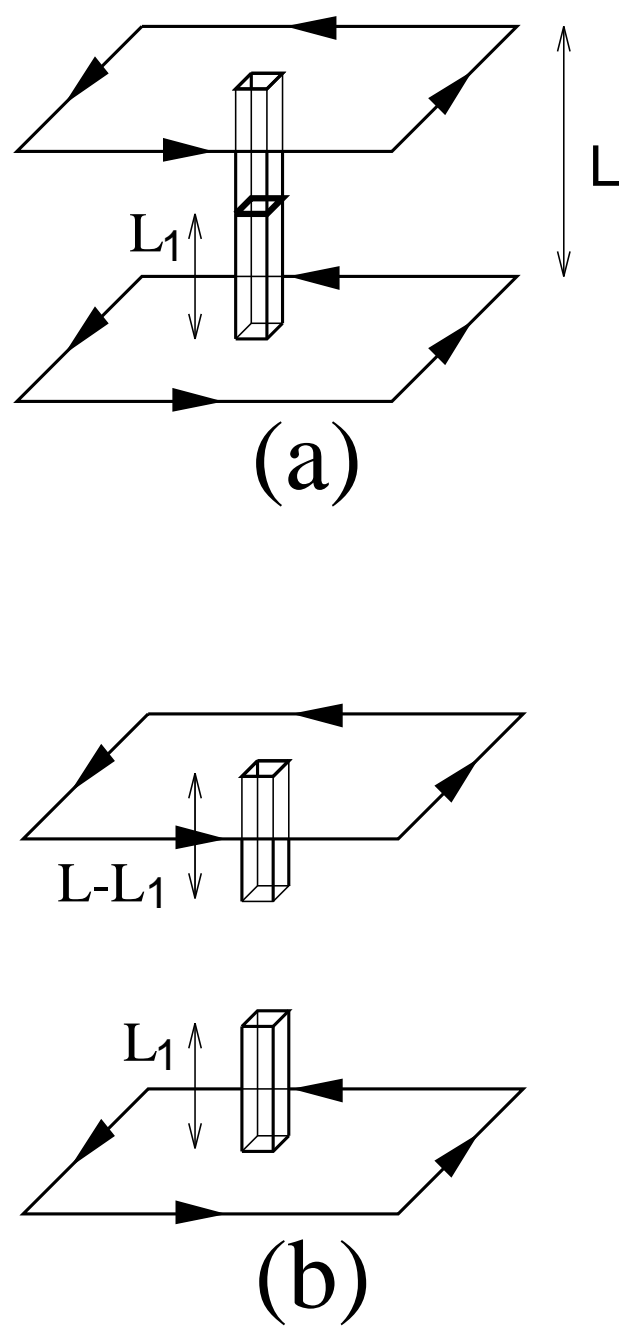

Figure 3 


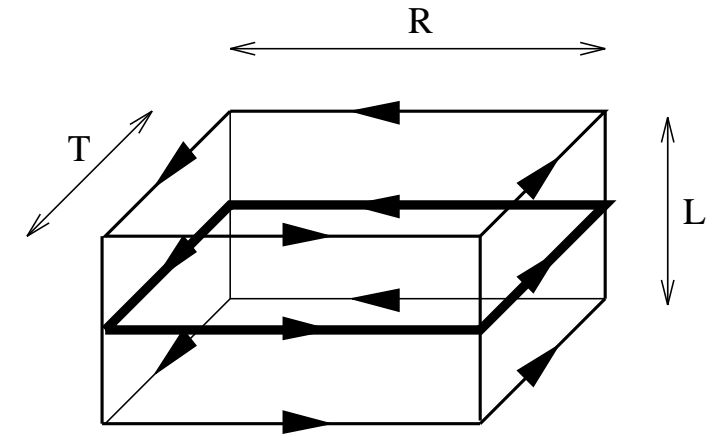

Figure 4 


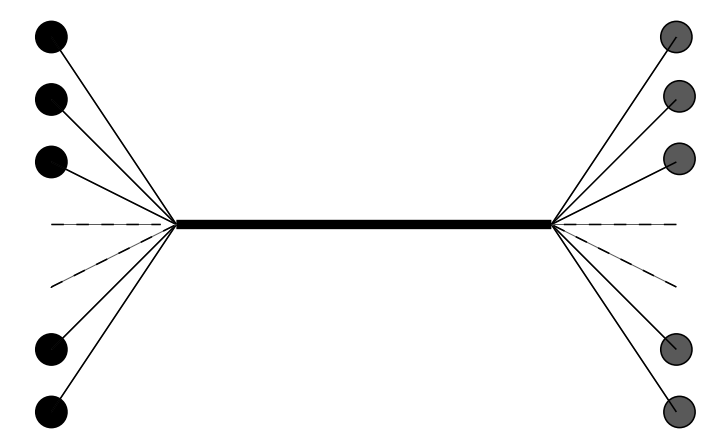

Figure 5 


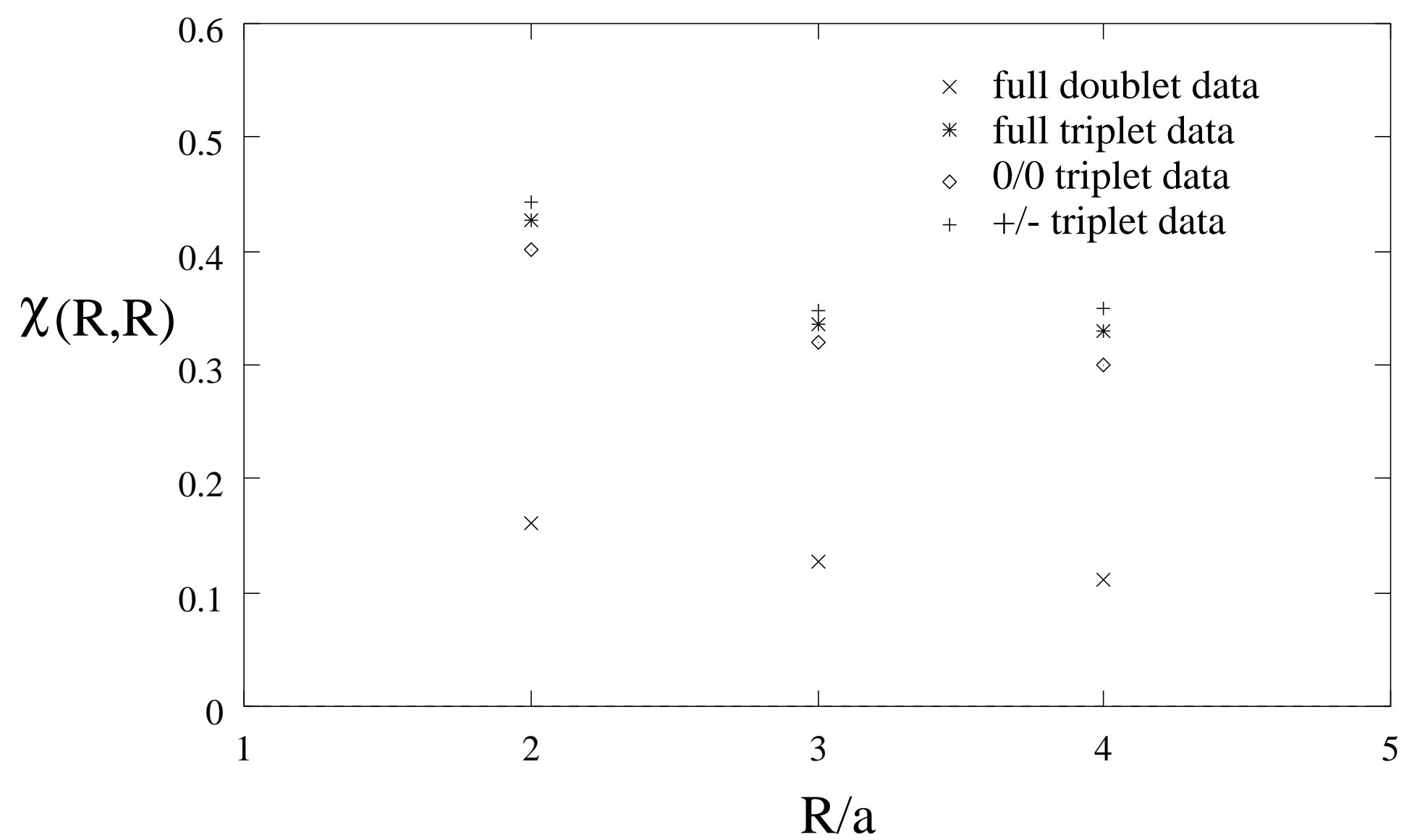

Figure 6 


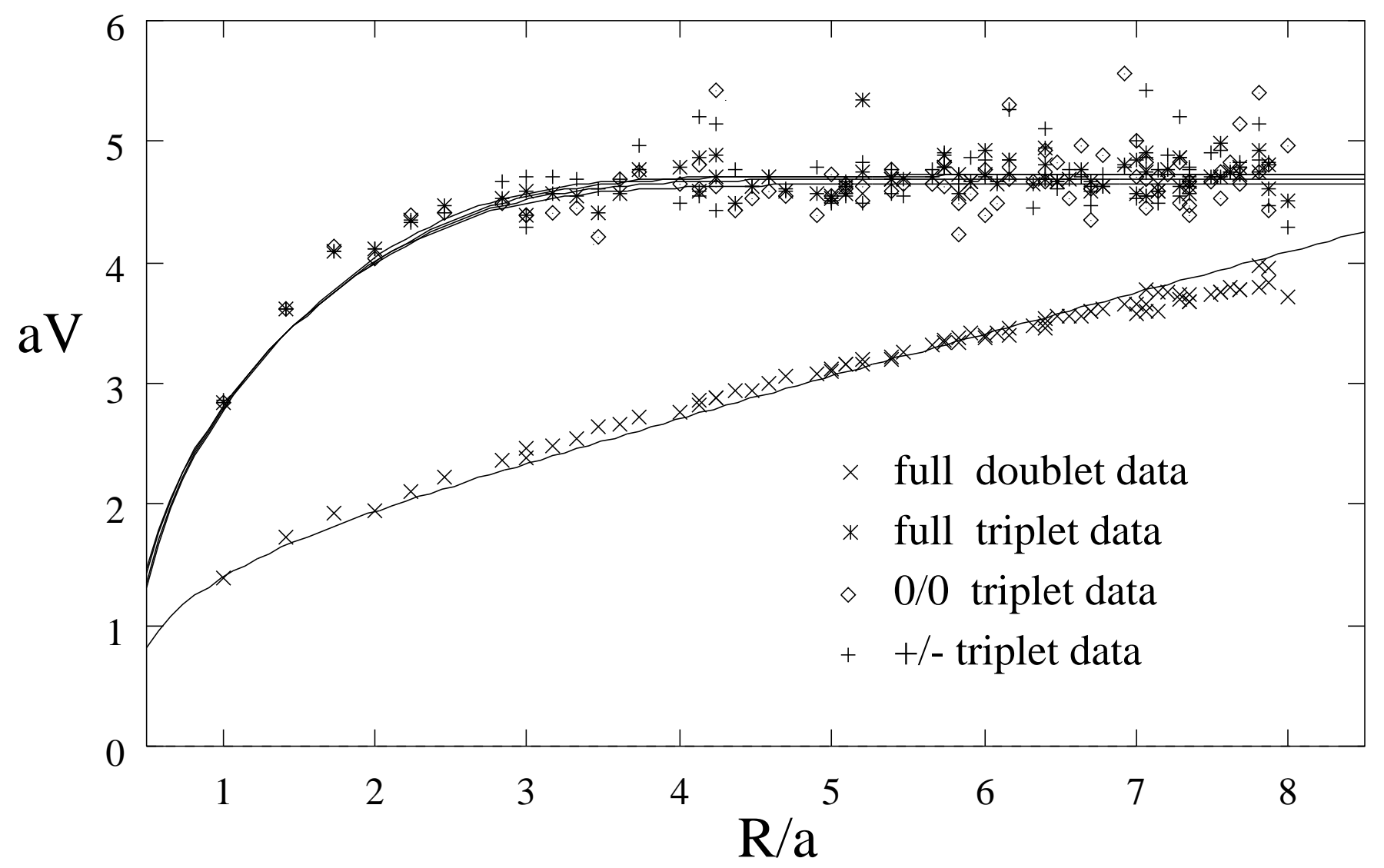

Figure 7 


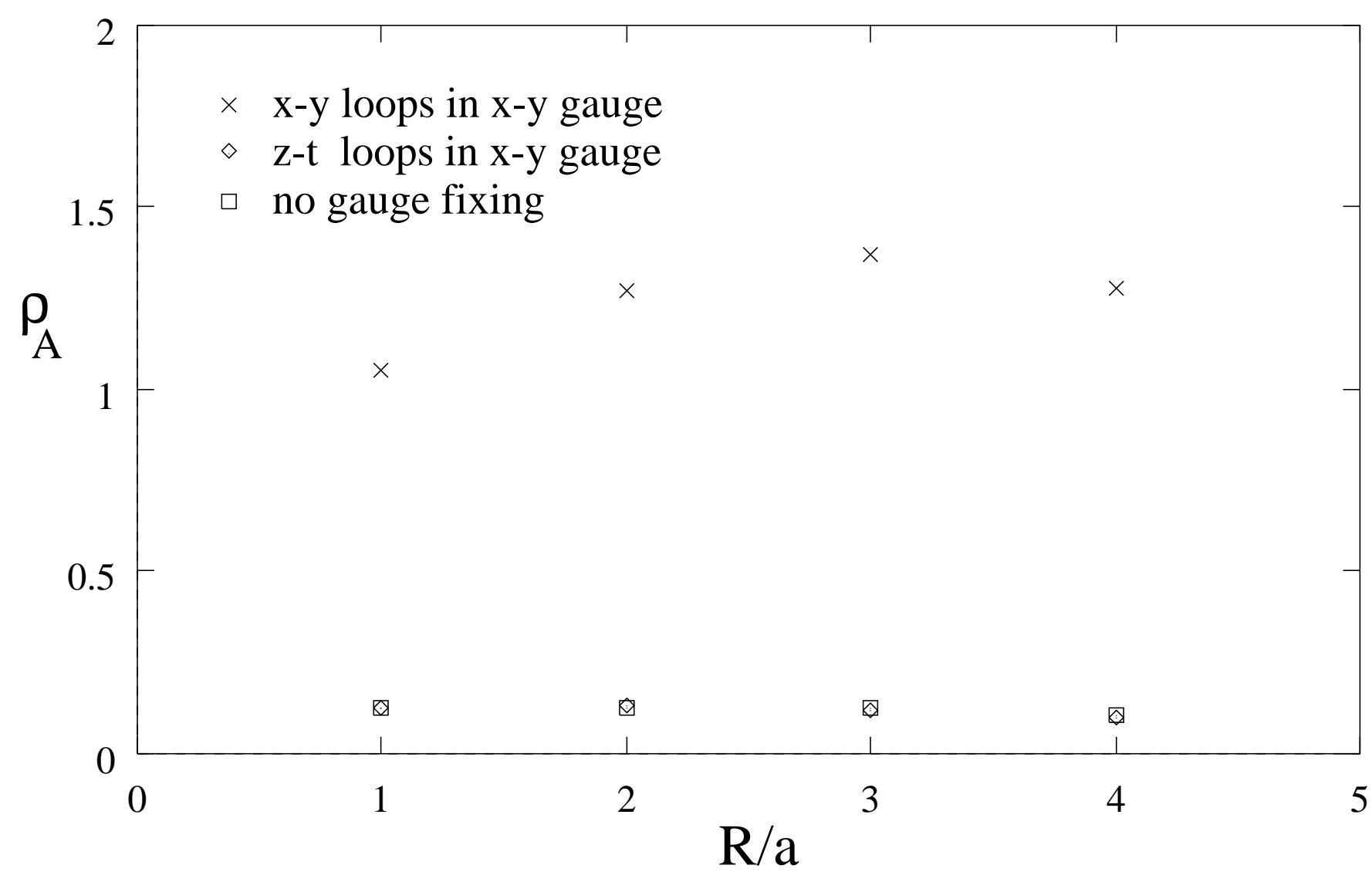

Figure 8 